\title{
On a fourth order elliptic equation with supercritical exponent
}

\section{Kamal Ould Bouh*}

\section{"Correspondence:}

hbouh@taibahu.edu.sa;

kamal.bouh@gmail.com

Department of Mathematics, Taibah

University, P.O. Box 30002,

Almadinah Almunawwarah,

Kingdom of Saudi Arabia

\begin{abstract}
This paper is concerned with the semi-linear elliptic problem involving nearly critical exponent $\left(P_{\varepsilon}\right): \Delta^{2} u=|u|^{8 /(n-4)+\varepsilon} u$ in $\Omega, \Delta u=u=0$ on $\partial \Omega$, where $\Omega$ is a smooth bounded domain in $\mathbb{R}^{n}, n \geq 5$, and $\varepsilon$ is a positive real parameter. We show that, for $\varepsilon$ small, $\left(P_{\varepsilon}\right)$ has no sign-changing solutions with low energy which blow up at exactly three points. Moreover, we prove that $\left(P_{\varepsilon}\right)$ has no bubble-tower sign-changing solutions.
\end{abstract}

MSC: $35 J 20 ; 35 J 60$

Keywords: nonlinear problem; critical exponent; sign-changing solutions; bubble-tower solution

\section{Introduction and results}

We consider the following semi-linear elliptic problem with supercritical nonlinearity:

$$
\left(P_{\varepsilon}\right) \quad\left\{\begin{array}{l}
\Delta^{2} u=|u|^{p-1+\varepsilon} u \text { in } \Omega \\
\Delta u=u=0 \quad \text { on } \partial \Omega
\end{array}\right.
$$

where $\Omega$ is a smooth bounded domain in $\mathbb{R}^{n}, n \geq 5, \varepsilon$ is a positive real parameter and $p+1=\frac{2 n}{n-4}$ is the critical Sobolev exponent for the embedding of $H^{2}(\Omega) \cap H_{0}^{1}(\Omega)$ into $L^{p+1}(\Omega)$.

When the biharmonic operator in $\left(P_{\varepsilon}\right)$ is replaced by the Laplacian operator, there are many works devoted to the study of the counterpart of $\left(P_{\varepsilon}\right)$; see for example [1-6], and the references therein.

When $\varepsilon<0$, many works have been devoted to the study of the solutions of $\left(P_{\varepsilon}\right)$ see for example [7-9]. In the critical case, this problem is not compact, that is, when $\varepsilon=0$ it corresponds exactly to the limiting case of the Sobolev embedding $H^{2}(\Omega) \cap H_{0}^{1}(\Omega)$ into $L^{p+1}(\Omega)$, and thus we lose the compact embedding. In fact, van Der Vorst showed in [10] that $\left(P_{0}\right)$ has no positive solutions if $\Omega$ is a starshaped domain. Whereas Ebobisse and Ould Ahmedou proved in [11] that $\left(P_{0}\right)$ has a positive solution provided that some homology group of $\Omega$ is non-trivial. This topological condition is sufficient, but not necessary, as examples of contractible domains $\Omega$ on which a positive solution exists show [12].

In the supercritical case, $\varepsilon>0$, the problem $\left(P_{\varepsilon}\right)$ becomes more delicate since we lose the Sobolev embedding which is an important point to overcome. The problem $\left(P_{\varepsilon}\right)$ was studied in [7] where the authors show that there is no one-bubble solution to the problem

\section{黛 Springer}

( 2014 Ould Bouh; licensee Springer. This is an Open Access article distributed under the terms of the Creative Commons Attribution License (http://creativecommons.org/licenses/by/2.0), which permits unrestricted use, distribution, and reproduction in any medium, provided the original work is properly cited. 
and there is a one-bubble solution to the slightly subcritical case under some suitable conditions. However, we proved in [13] that $\left(P_{\varepsilon}\right)$ has no sign-changing solutions which blow up exactly at two points. In this work we will show the non-existence of sign-changing solutions of $\left(P_{\varepsilon}\right)$ having three concentration points.

We note that problem $\left(P_{\varepsilon}\right)$ has a variational structure. The related functional is

$$
\inf J(u), \quad \text { where } J(u):=\frac{\int_{\Omega}|\Delta u|^{2}}{\left(\int_{\Omega}|u|^{p+1+\varepsilon}\right)^{2 /(p+1+\varepsilon)}}, u \in H^{2}(\Omega) \cap H_{0}^{1}(\Omega), u \neq 0 .
$$

$J$ satisfies the Palais-Smale condition in the subcritical case, while this condition fails in the critical case. Such a failure is due to the functions

$$
\delta_{(a, \lambda)}(x)=c_{0} \frac{\lambda^{(n-4) / 2}}{\left(1+\lambda^{2}|x-a|^{2}\right)^{(n-4) / 2}}, \quad c_{0}=\left(n(n-4)\left(n^{2}-4\right)\right)^{(n-4) / 8}, \lambda>0, a \in \mathbb{R}^{n} .
$$

$c_{0}$ is chosen so that $\delta_{(a, \lambda)}$ is the family of solutions of the following problem:

$$
\Delta^{2} u=u^{p}, \quad u>0 \text { in } \mathbb{R}^{n} .
$$

When we study problem (1.2) in a bounded smooth domain $\Omega$, we need to introduce the function $P \delta_{(a, \lambda)}$ which is the projection of $\delta_{(a, \lambda)}$ on $H_{0}^{1}(\Omega)$. It satisfies

$$
\Delta^{2} P \delta_{(a, \lambda)}=\Delta^{2} \delta_{(a, \lambda)} \quad \text { in } \Omega, \quad \Delta P \delta_{(a, \lambda)}=P \delta_{(a, \lambda)}=0 \quad \text { on } \partial \Omega
$$

These functions are almost positive solutions of (1.2).

We denote by $G$ the Green's function defined by, $\forall x \in \Omega$,

$$
\Delta^{2} G(x, \cdot)=c_{n} \delta_{x} \quad \text { in } \Omega, \quad \Delta G(x, \cdot)=G(x, \cdot)=0 \quad \text { on } \partial \Omega,
$$

where $\delta_{x}$ is the Dirac mass at $x$ and $c_{n}=(n-4)(n-2) w_{n}$, with $w_{n}$ is the area of the unit sphere of $\mathbb{R}^{n}$. We denote by $H$ the regular part of $G$, that is,

$$
H\left(x_{1}, x_{2}\right)=\left|x_{1}-x_{2}\right|^{4-n}-G\left(x_{1}, x_{2}\right) \quad \text { for }\left(x_{1}, x_{2}\right) \in \Omega^{2} .
$$

For $x=\left(x_{1}, x_{2}\right) \in \Omega^{2} \backslash \Gamma$, with $\Gamma=\{(y, y): y \in \Omega\}$, we denote by $M(x)$ the matrix defined by

$$
M(x)=\left(m_{i j}\right)_{1 \leq i, j \leq 2}, \quad \text { where } m_{i i}=H\left(x_{i}, x_{i}\right), m_{12}=m_{21}=G\left(x_{1}, x_{2}\right),
$$

and let $\rho(x)$ be its least eigenvalue.

The space $H^{2}(\Omega) \cap H_{0}^{1}(\Omega)$ is equipped with the norm $\|\cdot\|$ and its corresponding inner product $\langle\cdot, \cdot\rangle$ defined by

$$
\|u\|=\left(\int_{\Omega}|\Delta u|^{2}\right)^{1 / 2} \text { and }\langle u, v\rangle=\int_{\Omega} \Delta u \Delta v, \quad u, v \in H^{2}(\Omega) \cap H_{0}^{1}(\Omega) .
$$

Now, we are able to state our result. 
Theorem 1.1 Let $\Omega$ be any smooth bounded domain in $\mathbb{R}^{n}, n \geq 6$. If 0 is a regular value of $\rho(x)$, then there exists $\varepsilon_{0}>0$, such that, for each $\varepsilon \in\left(0, \varepsilon_{0}\right)$, problem $\left(P_{\varepsilon}\right)$ has no signchanging solutions $u_{\varepsilon}$ which satisfy

$$
u_{\varepsilon}=P \delta_{\left(a_{\varepsilon, 1}, \lambda_{\varepsilon, 1}\right)}-P \delta_{\left(a_{\varepsilon, 2}, \lambda_{\varepsilon, 2}\right)}+P \delta_{\left(a_{\varepsilon, 3}, \lambda_{\varepsilon, 3}\right)}+v_{\varepsilon}
$$

with $\left|u_{\varepsilon}\right|_{\infty}^{\varepsilon}$ is bounded and

$$
\left\{\begin{array}{l}
a_{\varepsilon, i} \in \Omega, \quad \lambda_{\varepsilon, i} d\left(a_{\varepsilon, i}, \partial \Omega\right) \rightarrow \infty \text { for } i=1,2,3, \\
\left\langle P \delta_{\left(a_{\varepsilon, i}, \lambda_{\varepsilon, i}\right)}, P \delta_{\left(a_{\varepsilon, j}, \lambda_{\varepsilon, j}\right)}\right\rangle \rightarrow 0 \text { for } i \neq j \text { and }\left\|v_{\varepsilon}\right\| \rightarrow 0 \text { as } \varepsilon \rightarrow 0 .
\end{array}\right.
$$

The second result deals with the phenomenon of bubble-tower solutions for the biharmonic problem $\left(P_{\varepsilon}\right)$ with supercritical exponent. We will give a generalization of the result found in [13]. More precisely, we have the following.

Theorem 1.2 Let $\Omega$ be any smooth bounded domain in $\mathbb{R}^{n}, n \geq 5$. There exists $\varepsilon_{0}>0$, such that, for each $\varepsilon \in\left(0, \varepsilon_{0}\right)$, problem $\left(P_{\varepsilon}\right)$ has no solutions $u_{\varepsilon}$ of the form

$$
u_{\varepsilon}=\sum_{i=1}^{k} \gamma_{i} P \delta_{\left(a_{\varepsilon, i}, \lambda_{\varepsilon, i}\right)}+v_{\varepsilon}, \quad \text { with } \lambda_{\varepsilon, 1} \leq \lambda_{\varepsilon, 2} \leq \cdots \leq \lambda_{\varepsilon, k} \text { and }\left|u_{\varepsilon}\right|_{\infty}^{\varepsilon} \text { is bounded }
$$

where $k \geq 2, \gamma_{i} \in\{-1,1\}, a_{\varepsilon, i} \in \Omega$, for each $i \leq j, \lambda_{\varepsilon, i}\left|a_{\varepsilon, i}-a_{\varepsilon, j}\right|$ is bounded and as $\varepsilon \rightarrow 0$, $\left\|v_{\varepsilon}\right\| \rightarrow 0, \lambda_{\varepsilon, i} d\left(a_{\varepsilon, i}, \partial \Omega\right) \rightarrow+\infty,\left\langle P \delta_{\left(a_{\varepsilon, i}, \lambda_{\varepsilon, i}\right)}, P \delta_{\left(a_{\varepsilon, j}, \lambda_{\varepsilon, j}\right)}\right\rangle \rightarrow 0$ for $i \neq j$, and if $l \notin\{k-1, k\}$, $\lambda_{\varepsilon, l}\left|a_{\varepsilon, l}-a_{\varepsilon, l+1}\right| \rightarrow 0$, where $l=\min \left\{q: \gamma_{q}=\cdots=\gamma_{k}\right\}$.

The proof of our results will be by contradiction. Thus, throughout this paper we will assume that there exist solutions $\left(u_{\varepsilon}\right)$ of $\left(P_{\varepsilon}\right)$ which satisfy (1.5) or (1.6). In Section 2, we will obtain some information as regards such $\left(u_{\varepsilon}\right)$ which allows us to develop Section 3 which deals with some useful estimates to the proof of our theorems. Finally, in Section 4, we combine these estimates to obtain a contradiction. Hence the proof of our results follows.

\section{Preliminary results}

In this section, we assume that there exist solutions $\left(u_{\varepsilon}\right)$ of $\left(P_{\varepsilon}\right)$ which satisfy

$$
u_{\varepsilon}=\sum_{i=1}^{k} \gamma_{i} P \delta_{\left(a_{\varepsilon, i}, \lambda_{\varepsilon, i}\right)}+v_{\varepsilon}
$$

with $\left|u_{\varepsilon}\right|_{\infty}^{\varepsilon}$ is bounded, $k \geq 2, a_{\varepsilon, i} \in \Omega$, and as $\varepsilon \rightarrow 0,\left\|v_{\varepsilon}\right\| \rightarrow 0, \lambda_{\varepsilon, i} d\left(a_{\varepsilon, i}, \partial \Omega\right) \rightarrow+\infty$, $\left\langle P \delta_{\left(a_{\varepsilon, i}, \lambda_{\varepsilon, i}\right)}, P \delta_{\left(a_{\varepsilon, j}, \lambda_{\varepsilon, j}\right)}\right\rangle \rightarrow 0$ for $i \neq j$. Arguing as in [14] and [15], we see that for $u_{\varepsilon}$ satisfying (2.1), there is a unique way to choose $\alpha_{i}, a_{i}, \lambda_{i}$, and $v$ such that

$$
\begin{aligned}
& u_{\varepsilon}=\sum_{i=1}^{k} \gamma_{i} \alpha_{i} P \delta_{\left(a_{i}, \lambda_{i}\right)}+v, \\
& \text { with } \begin{cases}\alpha_{i} \in \mathbb{R}, & \alpha_{i} \rightarrow 1, \\
a_{i} \in \Omega, & \lambda_{i} \in \mathbb{R}_{+}^{*}, \quad \lambda_{i} d\left(a_{i}, \partial \Omega\right) \rightarrow+\infty, \\
\nu \rightarrow 0 & \text { in } H^{2}(\Omega) \cap H_{0}^{1}(\Omega), \quad v \in E,\end{cases}
\end{aligned}
$$


where $E$ denotes the subspace of $H_{0}^{1}(\Omega)$ defined by

$$
E:=\left\{w:\langle w, \varphi\rangle=0, \forall \varphi \in \operatorname{Span}\left\{P \delta_{i}, \partial P \delta_{i} / \partial \lambda_{i}, \partial P \delta_{i} / \partial a_{i}^{j}, i \leq k ; j \leq n\right\}\right\}
$$

Here, $a_{i}^{j}$ denotes the $j$ th component of $a_{i}$ and in the sequel, in order to simplify the notations, we set $\delta_{\left(a_{i}, \lambda_{i}\right)}=\delta_{i}$ and $P \delta_{\left(a_{i}, \lambda_{i}\right)}=P \delta_{i}$. We always assume that $u_{\varepsilon}$ (which satisfies (2.1)) is written as in (2.2) and (2.3) holds. From (2.1), it is easy to see that the following remark holds.

Lemma 2.1 [13] Let $u_{\varepsilon}$ satisfying the assumption of the theorems. $\lambda_{i}$ occurring in (2.2) satisfies

$$
\lambda_{i}^{\varepsilon} \rightarrow 1 \quad \text { as } \varepsilon \rightarrow 0 \text { for each } i \leq k .
$$

Remark 2.2 [2,16] We recall the following estimate:

$$
\delta_{i}^{\varepsilon}(x)-c_{0}^{\varepsilon} \lambda_{i}^{\varepsilon(n-4) / 2}=O\left(\varepsilon \log \left(1+\lambda_{i}^{2}\left|x-a_{i}\right|^{2}\right)\right) \quad \text { in } \Omega .
$$

\section{Some useful estimates}

As usual in this type of problems, we first deal with the $v$-part of $u_{\varepsilon}$, in order to show that it is negligible with respect to the concentration phenomenon.

Lemma 3.1 The function $v$ defined in (2.2), satisfies the following estimate:

$$
\|v\| \leq c \varepsilon+c \begin{cases}\sum_{i} \frac{1}{\left(\lambda_{i} d_{i}\right)^{n-4}}+\sum_{i \neq j} \varepsilon_{i j}\left(\log \varepsilon_{i j}^{-1}\right)^{(n-4) / n} & \text { if } n<12, \\ \sum_{i} \frac{1}{\left(\lambda_{i} d_{i}\right)^{(n+4) / 2-\varepsilon(n-4)}}+\sum_{i \neq j} \varepsilon_{i j}^{(n+4) / 2(n-4)}\left(\log \varepsilon_{i j}^{-1}\right)^{(n+4) / 2 n} & \text { if } n \geq 12,\end{cases}
$$

where $d_{i}:=d\left(a_{i}, \partial \Omega\right)$ for $i \leq k$ and for $i \neq j, \varepsilon_{i j}$ is defined by

$$
\varepsilon_{i j}=\left(\frac{\lambda_{i}}{\lambda_{j}}+\frac{\lambda_{j}}{\lambda_{i}}+\lambda_{i} \lambda_{j}\left|a_{i}-a_{j}\right|^{2}\right)^{(4-n) / 2}
$$

Proof The proof is the same as that of Lemma 3.1 of [13], so we omit it.

Now, we state the crucial points in the proof of our theorems.

Proposition 3.2 Assume that $n \geq 5$ and let $\alpha_{i}, a_{i}$ and $\lambda_{i}$ be the variables defined in (2.2) with $k=3$ and $\gamma_{1}=-\gamma_{2}=\gamma_{3}$. We have

$$
\begin{aligned}
& \left|\alpha_{i} c_{1} \frac{n-4}{2} \frac{H\left(a_{i}, a_{i}\right)}{\lambda_{i}^{n-4}}+\sum_{j \neq i}(-1)^{i+j} \alpha_{j} c_{1}\left(\lambda_{i} \frac{\partial \varepsilon_{i j}}{\partial \lambda_{i}}+\frac{n-4}{2} \frac{H\left(a_{i}, a_{j}\right)}{\left(\lambda_{i} \lambda_{j}\right)^{(n-4) / 2}}\right)+\alpha_{i} \frac{n-4}{2} c_{2} \varepsilon\right| \\
& \leq c \varepsilon^{2}+c \begin{cases}\sum_{k} \frac{1}{\left(\lambda_{k} d_{k}\right)^{n-2}}+\sum_{j \neq i}\left(\varepsilon_{i j}^{\frac{n}{n-4}} \log \varepsilon_{i j}^{-1}+\varepsilon_{i j}^{2}\left(\log \varepsilon_{12}^{-1}\right)^{\frac{2(n-4)}{n}}\right) & \text { if } n \geq 6, \\
\sum_{k} \frac{1}{\left(\lambda_{k} d_{k}\right)^{2}}+\sum_{j \neq i} \varepsilon_{i j}^{2}\left(\log \varepsilon_{12}^{-1}\right)^{2 / 5} & \text { if } n=5,\end{cases}
\end{aligned}
$$

where $i, j \in\{1,2,3\}$ with $i \neq j$ and $c_{1}, c_{2}$ are positive constants. 
Proof Let

$$
c_{1}=c_{0}^{\frac{2 n}{n-4}} \int_{\mathbb{R}^{n}} \frac{d x}{\left(1+|x|^{2}\right)^{(n+4) / 2}}
$$

and

$$
c_{2}=\frac{n-4}{2} c_{0}^{\frac{2 n}{n-4}} \int_{\mathbb{R}^{n}} \log \left(1+|x|^{2}\right) \frac{|x|^{2}-1}{\left(1+|x|^{2}\right)^{n+1}} d x .
$$

It suffices to prove the proposition for $i=1$. Multiplying $\left(P_{\varepsilon}\right)$ by $\lambda_{1} \partial P \delta_{1} / \partial \lambda_{1}$ and integrating on $\Omega$, we obtain

$$
\begin{aligned}
& \alpha_{1} \int_{\Omega} \delta_{1}^{p} \lambda_{1} \frac{\partial P \delta_{1}}{\partial \lambda_{1}}-\alpha_{2} \int_{\Omega} \delta_{2}^{p} \lambda_{1} \frac{\partial P \delta_{1}}{\partial \lambda_{1}}+\alpha_{3} \int_{\Omega} \delta_{3}^{p} \lambda_{1} \frac{\partial P \delta_{1}}{\partial \lambda_{1}} \\
& \quad=\int_{\Omega}\left|u_{\varepsilon}\right|^{p-1+\varepsilon} u_{\varepsilon} \lambda_{1} \frac{\partial P \delta_{1}}{\partial \lambda_{1}} .
\end{aligned}
$$

Using [17], we derive

$$
\begin{aligned}
& \int_{\Omega} \delta_{1}^{p} \lambda_{1} \frac{\partial P \delta_{1}}{\partial \lambda_{1}}=\frac{n-4}{2} c_{1} \frac{H\left(a_{1}, a_{1}\right)}{\lambda_{1}^{n-4}}+O\left(\frac{\log \left(\lambda_{1} d_{1}\right)}{\left(\lambda_{1} d_{1}\right)^{n-1}}\right), \\
& \int_{\Omega} \delta_{j}^{p} \lambda_{1} \frac{\partial P \delta_{1}}{\partial \lambda_{1}}=c_{1}\left(\lambda_{1} \frac{\partial \varepsilon_{1 j}}{\partial \lambda_{1}}+\frac{n-4}{2} \frac{H\left(a_{1}, a_{j}\right)}{\left(\lambda_{1} \lambda_{j}\right)^{(n-4) / 2}}\right)+R_{j},
\end{aligned}
$$

where $j=2,3$ and $R_{j}$ satisfies

$$
R_{j}=O\left(\sum_{k=1, j} \frac{\log \left(\lambda_{k} d_{k}\right)}{\left(\lambda_{k} d_{k}\right)^{n-1}}+\varepsilon_{1 j}^{\frac{n}{n-4}} \log \varepsilon_{1 j}^{-1}\right) .
$$

For the other term of (3.3), we have

$$
\begin{aligned}
& \int_{\Omega}\left|u_{\varepsilon}\right|^{p-1+\varepsilon} u_{\varepsilon} \lambda_{1} \frac{\partial P \delta_{1}}{\partial \lambda_{1}} \\
& =\int_{\Omega}\left|\alpha_{1} P \delta_{1}-\alpha_{2} P \delta_{2}+\alpha_{3} P \delta_{3}\right|^{p-1+\varepsilon}\left(\alpha_{1} P \delta_{1}-\alpha_{2} P \delta_{2}+\alpha_{3} P \delta_{3}\right) \lambda_{1} \frac{\partial P \delta_{1}}{\partial \lambda_{1}} \\
& \quad+(p+\varepsilon) \int_{\Omega}\left|\alpha_{1} P \delta_{1}-\alpha_{2} P \delta_{2}+\alpha_{3} P \delta_{3}\right|^{p-1+\varepsilon} v \lambda_{1} \frac{\partial P \delta_{1}}{\partial \lambda_{1}} \\
& \quad+O\left(\|v\|^{2}+\sum_{i \neq j} \varepsilon_{i j}^{\frac{n}{n-4}} \log \varepsilon_{i j}^{-1}\right) .
\end{aligned}
$$

Concerning the last integral, it can be written as

$$
\begin{aligned}
& \int_{\Omega}\left|\alpha_{1} P \delta_{1}-\alpha_{2} P \delta_{2}+\alpha_{3} P \delta_{3}\right|^{p-1+\varepsilon} v \lambda_{1} \frac{\partial P \delta_{1}}{\partial \lambda_{1}} \\
& =\int_{\Omega}\left(\alpha_{1} P \delta_{1}\right)^{p-1+\varepsilon} v \lambda_{1} \frac{\partial P \delta_{1}}{\partial \lambda_{1}}+O\left(\int_{\Omega \backslash A_{j}} P \delta_{j}^{p-1} P \delta_{1}|v|+\int_{A_{j}} P \delta_{1}^{p-1} P \delta_{2}|v|\right),
\end{aligned}
$$

where $A_{j}=\left\{x: 2 \alpha_{j} P \delta_{j} \leq \alpha_{1} P \delta_{1}\right\}$ for $j=2,3$. 
Observe that, for $n \geq 12$, we have $p-1=8 /(n-4) \leq 1$, thus

$$
\begin{aligned}
\int_{\Omega \backslash A_{j}} P \delta_{j}^{p-1} P \delta_{1}|v|+\int_{A_{j}} P \delta_{1}^{p-1} P \delta_{j}|v| & \leq c \int_{\Omega}|v|\left(\delta_{1} \delta_{j}\right)^{\frac{n+4}{2(n-4)}} \\
& \leq c\|v\| \varepsilon_{1 j}^{(n+4) / 2(n-4)}\left(\log \varepsilon_{1 j}^{-1}\right)^{(n+4) / 2 n} .
\end{aligned}
$$

But for $n<12$, we have

$$
\int_{\Omega \backslash A_{j}} P \delta_{j}^{p-1} P \delta_{1}|v|+\int_{A} P \delta_{1}^{p-1} P \delta_{j}|v| \leq c \varepsilon_{1 j}\left(\log \varepsilon_{1 j}^{-1}\right)^{(n-4) / n}\|v\| .
$$

For the other integral in $(3.8)$, using $[16,17]$, and Remark 2.2 , we get

$$
\begin{aligned}
& \int_{\Omega} P \delta_{1}^{p-1+\varepsilon} v \lambda_{1} \frac{\partial P \delta_{1}}{\partial \lambda_{1}} \\
& \quad=O\left(\|v\|\left[\varepsilon+\left(\frac{1}{\left(\lambda_{1} d_{1}\right)^{\inf (n-4,(n+4) / 2)}}(\text { if } n \neq 12)+\frac{\log \left(\lambda_{1} d_{1}\right)}{\left(\lambda_{1} d_{1}\right)^{4}}(\text { if } n=12)\right)\right]\right) .
\end{aligned}
$$

It remains to estimate the second integral of (3.7). We have

$$
\begin{aligned}
& \int_{\Omega}\left|\alpha_{1} P \delta_{1}-\alpha_{2} P \delta_{2}+\alpha_{3} P \delta_{3}\right|^{p-1+\varepsilon}\left(\alpha_{1} P \delta_{1}-\alpha_{2} P \delta_{2}+\alpha_{3} P \delta_{3}\right) \lambda_{1} \frac{\partial P \delta_{1}}{\partial \lambda_{1}} \\
& =\int_{\Omega}\left(\alpha_{1} P \delta_{1}\right)^{p+\varepsilon} \lambda_{1} \frac{\partial P \delta_{1}}{\partial \lambda_{1}}-\int_{\Omega}\left(\alpha_{2} P \delta_{2}\right)^{p+\varepsilon} \lambda_{1} \frac{\partial P \delta_{1}}{\partial \lambda_{1}}+\int_{\Omega}\left(\alpha_{3} P \delta_{3}\right)^{p+\varepsilon} \lambda_{1} \frac{\partial P \delta_{1}}{\partial \lambda_{1}} \\
& \quad-(p+\varepsilon)\left(\int_{\Omega} \alpha_{2} P \delta_{2}\left(\alpha_{1} P \delta_{1}\right)^{p-1+\varepsilon} \lambda_{1} \frac{\partial P \delta_{1}}{\partial \lambda_{1}}-\int_{\Omega} \alpha_{3} P \delta_{3}\left(\alpha_{1} P \delta_{1}\right)^{p-1+\varepsilon} \lambda_{1} \frac{\partial P \delta_{1}}{\partial \lambda_{1}}\right) \\
& \quad+O\left(\sum \varepsilon_{1 j}^{\frac{n}{n-4}} \log \varepsilon_{1 j}^{-1}\right) .
\end{aligned}
$$

Now, using Remark 2.2 and [17], we have

$$
\begin{aligned}
& \int_{\Omega} P \delta_{1}^{p+\varepsilon} \lambda_{1} \frac{\partial P \delta_{1}}{\partial \lambda_{1}}= \frac{n-4}{2}\left(c_{2} \varepsilon+2 c_{1} \frac{H\left(a_{1}, a_{1}\right)}{\lambda_{1}^{n-4}}\right) \\
&+O\left(\varepsilon^{2}+\frac{\log \left(\lambda_{1} d_{1}\right)}{\left(\lambda_{1} d_{1}\right)^{n-1}}+\frac{1}{\left(\lambda_{1} d_{1}\right)^{2}}(\text { if } n=5)\right), \\
& \int_{\Omega} P \delta_{j}^{p+\varepsilon} \lambda_{1} \frac{\partial P \delta_{1}}{\partial \lambda_{1}}= c_{1}\left(\lambda_{1} \frac{\partial \varepsilon_{1 j}}{\partial \lambda_{1}}+\frac{n-4}{2} \frac{H\left(a_{1}, a_{j}\right)}{\left(\lambda_{1} \lambda_{j}\right)^{(n-4) / 2}}\right)+T_{j}, \\
& p \int_{\Omega} P \delta_{j} P \delta_{1}^{p-1+\varepsilon} \lambda_{1} \frac{\partial P \delta_{1}}{\partial \lambda_{1}}=c_{1}\left(\lambda_{1} \frac{\partial \varepsilon_{1 j}}{\partial \lambda_{1}}+\frac{n-4}{2} \frac{H\left(a_{1}, a_{j}\right)}{\left(\lambda_{1} \lambda_{j}\right)^{(n-4) / 2}}\right)+T_{j},
\end{aligned}
$$

where for $i=2,3$,

$$
\begin{aligned}
T_{i}= & O\left(\varepsilon \varepsilon_{1 j}\left(\log \varepsilon_{1 j}^{-1}\right)^{\frac{n-4}{n}}\right)+\left(\varepsilon_{1 j}^{\frac{n}{n-4}}\left(\log \varepsilon_{1 j}^{-1}\right)+\frac{\log \left(\lambda_{i} d_{i}\right)}{\left(\lambda_{i} d_{i}\right)^{n}}(\text { if } n \geq 8)\right) \\
& +\left(\frac{\varepsilon_{1 j}\left(\log \varepsilon_{1 j}^{-1}\right)^{\frac{n-4}{n}}}{\left(\lambda_{i} d_{i}\right)^{n-4}}(\text { if } n<8)\right) .
\end{aligned}
$$

Therefore, combining (3.3)-(3.15), and Lemma 3.1, the proof of Proposition 3.2 follows. 
Proposition 3.3 Let $n \geq 6$. We have the following estimate:

$$
\begin{aligned}
& \alpha_{i} \frac{1}{\lambda_{i}^{n-3}} \frac{\partial H\left(a_{i}, a_{i}\right)}{\partial a_{i}}-\frac{2}{\lambda_{i}} \sum_{j \neq i}(-1)^{i+j} \alpha_{j}\left(\frac{\partial \varepsilon_{i j}}{\partial a_{i}}-\frac{1}{\left(\lambda_{i} \lambda_{j}\right)^{(n-4) / 2}} \frac{\partial H}{\partial a_{i}}\left(a_{i}, a_{j}\right)\right) \\
& \quad=O\left(\sum_{k} \frac{1}{\left(\lambda_{k} d_{k}\right)^{n-2}}+\sum_{j \neq i} \varepsilon_{i j}^{\frac{n}{n-4}} \log \varepsilon_{i j}^{-1}+\varepsilon_{i j}^{2}\left(\log \varepsilon_{1 j}^{-1}\right)^{\frac{2(n-4)}{n}}+\varepsilon^{2}+\frac{\varepsilon}{\left(\lambda_{i} d_{i}\right)^{n-3}}\right),
\end{aligned}
$$

where $i, j \in\{1,2,3\}$ and $j \neq i$.

Proof The proof is similar to the proof of Proposition 3.2. But there exist some integrals which have different estimates. We will focus in those integrals. In fact, (3.3), (3.7)-(3.12) are also true if we change $\lambda_{1} \partial P \delta_{1} / \partial \lambda_{1}$ by $\left(1 / \lambda_{1}\right) \partial P \delta_{1} / \partial a_{1}$. It remains to deal with the other equations. Following [17], we get

$$
\begin{aligned}
& \int_{\Omega} \delta_{1}^{p} \frac{1}{\lambda_{1}} \frac{\partial P \delta_{1}}{\partial a_{1}}=-\frac{1}{2} \frac{c_{1}}{\lambda_{1}^{n-3}} \frac{\partial H\left(a_{1}, a_{1}\right)}{\partial a_{1}}+O\left(\frac{1}{\left(\lambda_{1} d_{1}\right)^{n-1}}\right) \\
& \int_{\Omega} \delta_{j}^{p} \frac{1}{\lambda_{1}} \frac{\partial P \delta_{1}}{\partial a_{1}}=\frac{c_{1}}{\lambda_{1}}\left(\frac{\partial \varepsilon_{1 j}}{\partial a_{1}}-\frac{1}{\left(\lambda_{1} \lambda_{j}\right)^{(n-4) / 2}} \frac{\partial H}{\partial a_{1}}\left(a_{1}, a_{j}\right)\right) \\
& +O\left(\sum_{k=1, j} \frac{1}{\left(\lambda_{k} d_{k}\right)^{n-1}}+\lambda_{j}\left|a_{1}-a_{j}\right| \varepsilon_{1 j}^{(n-1) /(n-4)}\right), \\
& \int_{\Omega} P \delta_{1}^{p+\varepsilon} \frac{1}{\lambda_{1}} \frac{\partial P \delta_{1}}{\partial a_{1}}=-c_{0}^{\varepsilon} \lambda_{1}^{\varepsilon(n-4) / 2} \frac{c_{1}}{\lambda_{1}^{n-3}} \frac{\partial H\left(a_{1}, a_{1}\right)}{\partial a_{1}}+O\left(\frac{1}{\left(\lambda_{1} d_{1}\right)^{n-2}}+\frac{\varepsilon}{\left(\lambda_{1} d_{1}\right)^{n-3}}\right), \\
& \int_{\Omega} P \delta_{j}^{p+\varepsilon} \frac{1}{\lambda_{1}} \frac{\partial P \delta_{1}}{\partial \lambda_{1}}=c_{0}^{\varepsilon} \lambda_{j}^{\varepsilon(n-4) / 2}\left(P \delta_{j}, \frac{1}{\lambda_{1}} \frac{\partial P \delta_{1}}{\partial a_{1}}\right)+O\left(\varepsilon \varepsilon_{1 j}\left(\log \varepsilon_{1 j}^{-1}\right)^{(n-4) / n}\right)+T_{j}, \\
& \int_{\Omega} P \delta_{j} \frac{1}{\lambda_{1}} \frac{\partial\left(P \delta_{1}^{p+\varepsilon}\right)}{\partial a_{1}}=c_{0}^{\varepsilon} \lambda_{1}^{\varepsilon(n-4) / 2}\left(P \delta_{j}, \frac{1}{\lambda_{1}} \frac{\partial P \delta_{1}}{\partial a_{1}}\right)+O\left(\varepsilon \varepsilon_{1 j}\left(\log \varepsilon_{1 j}^{-1}\right)^{(n-4) / n}\right)+T_{j} .
\end{aligned}
$$

The proof of Proposition 3.3 is thereby completed.

\section{Proof of the theorems}

\section{Proof of Theorem 1.1}

Arguing by contradiction, let us assume that problem $\left(P_{\varepsilon}\right)$ has solutions $\left(u_{\varepsilon}\right)$ as stated in Theorem 1.1. Recall that $u_{\varepsilon}$ is written as

$$
u_{\varepsilon}=\alpha_{\varepsilon, 1} P \delta_{\left(a_{\varepsilon, 1}, \lambda_{\varepsilon, 1}\right)}-\alpha_{\varepsilon, 2} P \delta_{\left(a_{\varepsilon, 2}, \lambda_{\varepsilon, 2}\right)}+\alpha_{\varepsilon, 3} P \delta_{\left(a_{\varepsilon, 3}, \lambda_{\varepsilon, 3}\right)}+v_{\varepsilon},
$$

with $v_{\varepsilon}$ orthogonal to each $P \delta_{\left(a_{i}, \lambda_{i}\right)}$ and their derivatives with respect to $\lambda_{i}$ and $\left(a_{i}\right)_{k}$, where $\left(a_{i}\right)_{k}$ denotes the $k$ th component of $a_{i}$ (see (2.2) and (2.3)). For simplicity, we will write $\alpha_{i}:=\alpha_{\varepsilon, i}, \lambda_{i}:=\lambda_{\varepsilon, i}$, and $a_{i}:=a_{\varepsilon, i}$. From Proposition 3.2, for each $i=1,2,3$, with $\gamma_{1}=\gamma_{3}=1$, $\gamma_{2}=-1$. We have

$$
\begin{gathered}
\left(E_{i}\right) \quad c_{1} \frac{n-4}{2} \frac{H\left(a_{i}, a_{i}\right)}{\lambda_{i}^{n-4}}+\gamma_{i} c_{1} \sum_{j \neq i} \gamma_{j}\left(\lambda_{i} \frac{\partial \varepsilon_{i j}}{\partial \lambda_{i}}+\frac{n-4}{2} \frac{H\left(a_{i}, a_{j}\right)}{\left(\lambda_{i} \lambda_{j}\right)^{(n-4) / 2}}\right)+\frac{n-4}{2} c_{2} \varepsilon \\
=o\left(\varepsilon+\sum_{j=1}^{3} \frac{1}{\left(\lambda_{j} d_{j}\right)^{n-4}}+\sum_{r \neq j} \varepsilon_{r j}\right) .
\end{gathered}
$$


Furthermore, an easy computation shows that

$$
\begin{aligned}
& \lambda_{i} \frac{\partial \varepsilon_{i j}}{\partial \lambda_{i}}=-\frac{n-4}{2} \varepsilon_{i j}\left(1-2 \frac{\lambda_{j}}{\lambda_{i}} \varepsilon_{i j}^{2 / n-4}\right) \quad \text { for } i, j=1,2,3, j \neq i, \\
& -\lambda_{i} \frac{\partial \varepsilon_{i j}}{\partial \lambda_{i}}-2 \lambda_{j} \frac{\partial \varepsilon_{i j}}{\partial \lambda_{j}} \geq \frac{n-4}{2} \varepsilon_{i j} \text { for } \lambda_{i} \leq \lambda_{j} .
\end{aligned}
$$

On the other hand, following the proof of Proposition 3.3, we have, for each $i=1,2,3$,

$$
\begin{gathered}
\left(F_{i}\right) \frac{1}{\lambda_{i}^{n-3}} \frac{\partial H\left(a_{i}, a_{i}\right)}{\partial a_{i}}-\sum_{j \neq i} 2 \frac{(-1)^{j+i}}{\lambda_{i}}\left(\frac{\partial \varepsilon_{j i}}{\partial a_{i}}-\frac{\partial H\left(a_{j}, a_{i}\right)}{\partial a_{i}} \frac{1}{\left(\lambda_{j} \lambda_{i}\right)^{(n-4) / 2}}\right) \\
=o\left(\sum_{j} \frac{1}{\left(\lambda_{j} d_{j}\right)^{n-3}}+\sum_{r \neq j} \varepsilon_{r j}^{\frac{n-3}{n-4}}+\varepsilon^{\frac{n-3}{n-4}}\right) .
\end{gathered}
$$

We distinguish many cases depending on the set

$$
\digamma:=\left\{(i, j): i \neq j \text { and } \min \left(\lambda_{i}, \lambda_{j}\right)\left|a_{i}-a_{j}\right| \text { is bounded }\right\}
$$

and we will prove that all these cases cannot occur.

We remark that if $(i, j) \in \digamma$ we derive $\lambda_{i} / \lambda_{j} \rightarrow 0$ or $\infty$ and $d_{i} / d_{j}=1+o(1)$ as $\varepsilon \rightarrow 0$.

Furthermore, the behavior of $\varepsilon_{i j}$ depends on the set $\digamma$. In fact we have, assuming that $\lambda_{i} \leq \lambda_{j}$

$$
\begin{aligned}
& c\left(\frac{\lambda_{i}}{\lambda_{j}}\right)^{(n-4) / 2} \leq \varepsilon_{i j} \leq\left(\frac{\lambda_{i}}{\lambda_{j}}\right)^{(n-4) / 2} \quad \text { if }(i, j) \in \digamma, \\
& \varepsilon_{i j}=\frac{1}{\left(\lambda_{i} \lambda_{j}\left|a_{i}-a_{j}\right|^{2}\right)^{(n-4) / 2}}+o\left(\varepsilon_{i j}\right) \quad \text { if }(i, j) \notin \digamma .
\end{aligned}
$$

First we start by proving the following crucial lemmas.

Remark 4.1 Ordering the $\lambda_{i}{ }^{\prime} \mathrm{s}: \lambda_{i_{1}} \leq \lambda_{i_{2}} \leq \lambda_{i_{3}}$, adding $\left(E_{i_{1}}\right)+2\left(E_{i_{2}}\right)+4\left(E_{i_{3}}\right)$, and using (4.2), it is easy to derive a contradiction if we have $\varepsilon_{13}=o\left(\sum\left(\lambda_{i} d_{i}\right)^{4-n}+\sum \varepsilon_{r j}+\varepsilon\right)$.

Lemma 4.2 Let $n \geq 4$. Then there exists a positive constant $\underline{c}_{0}>0$ such that
(i) $\quad \underline{c}_{0}^{-1} \leq \frac{d_{1}}{d_{3}} \leq \underline{c}_{0}$;
(ii) $\underline{c}_{0}^{-1} \leq \frac{\lambda_{1}}{\lambda_{3}} \leq \underline{c}_{0}$;
(iii) $\quad \underline{c}_{0}^{-1} \leq \frac{\left|a_{1}-a_{3}\right|}{d_{i}} \leq \underline{c}_{0}^{-1} \quad$ for $i=1,3$.

Proof The proof will be by contradiction.

Proof of (i). Assume that $d_{1} / d_{3} \rightarrow 0$. In this case, we have

$$
\left|a_{1}-a_{3}\right| \geq c d_{3} \quad \text { and } \quad \varepsilon_{13}=\frac{1}{\left(\lambda_{1} \lambda_{3}\left|a_{1}-a_{3}\right|^{2}\right)^{(n-4) / 2}}+o\left(\varepsilon_{13}\right)
$$


which implies that $\varepsilon_{13}=o\left(\left(\lambda_{1} d_{1}\right)^{4-n}+\left(\lambda_{3} d_{3}\right)^{4-n}\right)$. Using Remark 4.1, we derive a contradiction. In the same way, we prove that $d_{3} / d_{1} \nrightarrow 0$. Hence the proof of Claim (i) is completed.

Proof of (ii). Assume that $\lambda_{1} / \lambda_{3} \rightarrow 0$. By Claim (i), we have $\left(\lambda_{3} d_{3}\right)^{-1}=o\left(\left(\lambda_{1} d_{1}\right)^{-1}\right)$. Four cases may occur.

Case 1. $\lambda_{2} / \lambda_{3} \nrightarrow 0$ or $\{(1,2),(2,3)\} \cap \digamma=\phi$. Using (4.5), $\left(E_{2}\right)$ implies that

$$
\frac{H\left(a_{2}, a_{2}\right)}{\lambda_{2}^{n-4}}+\varepsilon_{12}+\varepsilon_{23}+\varepsilon=o\left(\frac{1}{\left(\lambda_{1} d_{1}\right)^{n-4}}+\varepsilon_{13}\right)
$$

By Claim (i) and $\left(E_{3}\right)$, we obtain $\varepsilon_{13}=o\left(\left(\lambda_{1} d_{1}\right)^{4-n}\right)$. By Remark 4.1, this case cannot occur.

Case 2. $\lambda_{2} / \lambda_{3} \rightarrow 0,\{(1,2),(2,3)\} \cap \digamma \neq \phi$, and $\lambda_{2} / \lambda_{1} \rightarrow+\infty$. In this case, it is easy to obtain $\varepsilon_{13}=o\left(\varepsilon_{12}+\varepsilon_{23}\right)$. Using Remark 4.1, we derive a contradiction.

Case 3 . $\lambda_{2} / \lambda_{3} \rightarrow 0,(2,3) \in \digamma,(1,2) \notin \digamma$, and $\lambda_{2} / \lambda_{1} \nrightarrow+\infty$. In this case, we see that $\lambda_{2} \mid a_{2}-$ $a_{3} \mid$ is bounded and $\lambda_{2}\left|a_{1}-a_{2}\right| \rightarrow+\infty$. Hence, we derive that $\lambda_{2}\left|a_{1}-a_{3}\right| \rightarrow+\infty$, which implies that $\lambda_{k}\left|a_{1}-a_{3}\right| \rightarrow+\infty$ for $k=1,3$. Thus

$$
\varepsilon_{13}=\frac{1+o(1)}{\left(\lambda_{1} \lambda_{3}\left|a_{1}-a_{3}\right|^{2}\right)^{(n-4) / 2}}=\left(\frac{\lambda_{2}}{\lambda_{3}}\right)^{(n-4) / 2} \frac{1+o(1)}{\left(\lambda_{1} \lambda_{2}\left|a_{1}-a_{3}\right|^{2}\right)^{(n-4) / 2}}=o\left(\varepsilon_{23}\right)
$$

Then by Remark 4.1, we get a contradiction.

Case 4. $\lambda_{2} / \lambda_{3} \rightarrow 0,(1,2) \in \digamma$, and $\lambda_{2} / \lambda_{1} \nrightarrow+\infty$. In this case, it is easy to get $\varepsilon_{23}=o\left(\varepsilon_{12}\right)$. Using the formula $\left[\left(E_{1}\right)+\left(E_{2}\right)-\left(E_{3}\right)\right]$, we deduce that $\varepsilon=o\left(\varepsilon_{12}+\varepsilon_{13}\right)$, which implies that $\varepsilon_{13}=o\left(\varepsilon_{12}\right)$. Hence by Remark 4.1, we derive a contradiction and Claim (ii) is thereby completed.

Proof of (iii). Without loss of generality, we can assume that $d_{1} \leq d_{3}$. First, as in the proof of Claim (i), we get $\left|a_{1}-a_{3}\right| \leq c_{0} d_{1}$. Now assume that $\left|a_{1}-a_{3}\right| / d_{1} \rightarrow 0$, which implies

$$
\frac{H\left(a_{i}, a_{i}\right)}{\lambda_{i}^{n-4}}=o\left(\varepsilon_{13}\right) \quad \text { for } i=1,3
$$

Two cases may occur.

Case $1 . \lambda_{1} \leq \lambda_{2}$ or $\{(1,2),(2,3)\} \cap \digamma=\phi$. Using $\left(E_{2}\right)$, we obtain

$$
\frac{H\left(a_{2}, a_{2}\right)}{\lambda_{2}^{n-4}}=o\left(\varepsilon_{13}\right), \quad \varepsilon_{i 2}=o\left(\varepsilon_{13}\right) \quad \text { for } i=1,3 \text { and } \varepsilon=o\left(\varepsilon_{13}\right) \text {, }
$$

and we derive a contradiction from $\left(E_{1}\right)$.

Case 2. $\lambda_{2} \leq \lambda_{1}$ and $\{(1,2),(2,3)\} \cap \digamma \neq \phi$. Let $k \in\{1,3\}$ such that $(2, k) \in \digamma$. Using Claim (ii) and the fact that $\lambda_{2} \leq \lambda_{1}$, we derive that $\varepsilon_{2 k} \geq c\left(\lambda_{2} / \lambda_{k}\right)^{(n-4) / 2}$, which implies that $d_{2} \sim d_{k}, \lambda_{2} / \lambda_{k} \rightarrow 0$, and $\lambda_{2}\left|a_{2}-a_{k}\right|$ is bounded. Using (4.3) for $i=k$, we get

$$
-\lambda_{2}\left|a_{2}-a_{k}\right| \varepsilon_{2 k}^{\frac{n}{n-4}}+\frac{\lambda_{1} \lambda_{3}}{\lambda_{k}}\left|a_{1}-a_{3}\right| \varepsilon_{13}^{\frac{n}{n-4}}=o\left(\frac{1}{\left(\lambda_{2} d_{2}\right)^{n-3}}+\sum_{r \neq j} \varepsilon_{r j}^{\frac{n-3}{n-4}}+\varepsilon^{\frac{n-3}{n-4}}\right) .
$$

Since $\lambda_{2}\left|a_{2}-a_{k}\right|$ is bounded and $\varepsilon_{13} \simeq\left(\lambda_{1} \lambda_{3}\left|a_{2}-a_{k}\right|^{2}\right)^{(4-n) / 2}$, we derive that

$$
\varepsilon_{13}^{\frac{n-3}{n-4}}=o\left(\frac{1}{\left(\lambda_{2} d_{2}\right)^{n-3}}+\varepsilon_{12}^{\frac{n-3}{n-4}}+\varepsilon_{23}^{\frac{n-3}{n-4}}+\varepsilon^{\frac{n-3}{n-4}}\right)
$$


which implies that

$$
\varepsilon_{13}=o\left(\frac{1}{\left(\lambda_{2} d_{2}\right)^{n-4}}+\varepsilon_{12}+\varepsilon_{23}+\varepsilon\right) .
$$

By Remark 4.1, we get a contradiction.

Lemma 4.3 There exists a positive constant $\underline{c}_{0}^{\prime}$ such that

(i) $\underline{c}_{0}^{\prime} \lambda_{1} \leq \lambda_{2}$

(ii) $\quad d_{i} \geq \underline{c}_{0}^{\prime} \quad$ for $i=1,3$.

Proof Without loss of generality, we can assume that $d_{1} \leq d_{3}$.

Proof of (i). Assume that $\lambda_{2} / \lambda_{1} \rightarrow 0$. First we claim that $d_{1} / d_{2} \nrightarrow 0$. In fact, arguing by contradiction we assume that $d_{1} / d_{2} \rightarrow 0$, we get $d_{1} \rightarrow 0,\left|a_{1}-a_{2}\right| \geq c d_{2}$, and $\left|a_{2}-a_{3}\right| \geq c d_{2}$. Hence, $\{(1,2),(2,3)\} \cap \digamma=\phi$. From $\left(E_{2}\right)$, we obtain

$$
\frac{H\left(a_{2}, a_{2}\right)}{\lambda_{2}^{n-4}}+\varepsilon_{12}+\varepsilon_{23}+\varepsilon=o\left(\frac{1}{\left(\lambda_{1} d_{1}\right)^{n-4}}+\frac{1}{\left(\lambda_{3} d_{3}\right)^{n-4}}+\varepsilon_{13}\right) .
$$

Let $v_{i}$ be the outward normal vector at $a_{i}$. Since $d_{1}, d_{3}$, and $\left|a_{1}-a_{3}\right|$ are of the same order, we have (see [18] and [19])

$$
\frac{1}{\lambda_{1}^{n-3}} \frac{\partial H\left(a_{1}, a_{1}\right)}{\partial v_{1}} \sim \frac{c}{\left(\lambda_{1} d_{1}\right)^{n-3}} \quad \text { and } \quad \frac{\partial G\left(a_{1}, a_{3}\right)}{\partial v_{1}} \leq 0 .
$$

Using $\left(F_{1}\right)$, we get $1 /\left(\lambda_{1} d_{1}\right)^{n-3}=o\left(\varepsilon_{13}^{(n-3) /(n-4)}\right)$, which implies that $1 /\left(\lambda_{1} d_{1}\right)^{n-4}=o\left(\varepsilon_{13}\right)$. From $\left(E_{1}\right)$, we derive a contradiction. Hence our claim is proved.

Thus there exists a positive constant $c$ so that $d_{1} \geq c d_{2}$. Now, since we have assumed that $\lambda_{2} / \lambda_{1} \rightarrow 0$, Lemma 4.2 implies that $\varepsilon_{13}=o\left(\left(\lambda_{2} d_{2}\right)^{4-n}\right)$. Finally, using Remark 4.1, we get a contradiction and the proof of Claim (i) follows.

Proof of (ii). Assume that $d_{1} \rightarrow 0$. Note that Claim (i) and $\left(E_{2}\right)$ imply that (4.9) holds.

Now, following the proof of (i), we obtain a contradiction.

We turn now to the proof of Theorem 1.1. By the previous lemmas, we know that $\lambda_{1}$ and $\lambda_{3}$ are of the same order, $\left|a_{1}-a_{3}\right| \geq c$ and $\lambda_{2} \geq c \lambda_{i}$, for $i=1,3$ where $c$ is a positive constant. Hence, $\left(E_{2}\right)$ implies that (4.9) holds. Furthermore, for $i=1,3\left(E_{i}\right)$ implies that

$$
\frac{H\left(a_{i}, a_{i}\right)}{\lambda_{i}^{n-4}}-\frac{G\left(a_{1}, a_{3}\right)}{\left(\lambda_{1} \lambda_{3}\right)^{n-4}}=o\left(\frac{1}{\left(\lambda_{1} d_{1}\right)^{n-4}}+\frac{1}{\left(\lambda_{3} d_{3}\right)^{n-4}}+\varepsilon_{13}\right) .
$$

We denote by $r(x)$ the eigenvector associated to $\rho(x)$ whose norm is 1 . We point out that we can choose $r(x)$ so that all their components are positive (see [18] and [19]).

$$
\text { Let } \Lambda_{i}=\lambda_{i}^{(4-n) / 2}, \Lambda=\left(\Lambda_{1}, \Lambda_{3}\right) \text {, and } x=\left(a_{1}, a_{3}\right) \text {. From (4.11), we have }
$$

$$
M(x) \cdot \frac{{ }^{t} \Lambda}{\|\Lambda\|}=o(1)
$$


The scalar product of (4.12) by $r(x)$ gives

$$
\rho(x) r(x) \cdot \frac{{ }^{t} \Lambda}{\|\Lambda\|}=o(1)
$$

Since the components of $r(x)$ are positive and $\lambda_{1}, \lambda_{3}$ are of the same order, there exists a positive constant $c$, such that $r(x) \cdot \frac{t_{\Lambda}}{\|\Lambda\|} \geq c>0$. Hence, we get

$$
\rho(x)=o(1) .
$$

We deduce from (4.3) and (4.11) that

$$
\frac{\partial M}{\partial x_{i}}(x) \cdot \frac{{ }^{t} \Lambda}{\|\Lambda\|}=o(1)
$$

Observe that $\Lambda$ may be written in the form

$$
\Lambda=\beta r(x)+\bar{r}(x), \quad \text { with } r(x) \cdot \bar{r}(x)=0,\|\bar{r}\|=o(\beta) \text { and } \beta \sim\|\Lambda\| .
$$

Using (4.15), we get

$$
\frac{\partial M}{\partial x_{i}}(x) \cdot{ }^{t} r(x)+\frac{\partial M}{\partial x_{i}}(x) \cdot \frac{\bar{r}(x)}{\|\Lambda\|}=o(1) .
$$

Since $d_{i} \geq c_{0}$ for $i=1,3$ and $\left|a_{1}-a_{3}\right| \geq c_{0}$, the matrix $\frac{\partial M}{\partial x_{i}}(x)$ is bounded.

Furthermore, we have $\|\bar{r}\|=o(\|\Lambda\|)$, which implies that

$$
\frac{\partial M}{\partial x_{i}}(x) \cdot{ }^{t} r(x)=o(1)
$$

Let us consider the equality

$$
M(x) \cdot{ }^{t} r(x)=\rho(x) \cdot{ }^{t} r(x)
$$

and derivative it with respect to $x_{i}$; we obtain

$$
\frac{\partial M}{\partial x_{i}}(x) \cdot{ }^{t} r(x)+M(x) \frac{\partial^{t} r}{\partial x_{i}}(x)=\frac{\partial \rho}{\partial x_{i}}(x) \cdot{ }^{t} r(x)+\rho(x) \frac{\partial^{t} r}{\partial x_{i}}(x) .
$$

The scalar product with $r(x)$ gives

$$
r(x) \cdot \frac{\partial M}{\partial x_{i}}(x) \cdot{ }^{t} r(x)=\frac{\partial \rho}{\partial x_{i}}(x) .
$$

Using (4.18), we obtain

$$
\frac{\partial \rho}{\partial x_{i}}(x)=o(1) .
$$

Hence, we derive a contradiction from (4.14), (4.20), and the fact that 0 is a regular value of $\rho$. Thus the proof of our theorem follows. 


\section{Proof of Theorem 1.2}

Arguing by contradiction, let us assume that problem $\left(P_{\varepsilon}\right)$ has solutions $\left(u_{\varepsilon}\right)$ as stated in Theorem 1.2. From Section 2, these solutions have to satisfy (2.2) and (2.3).

As in the proof of Proposition 3.2, we have, for each $i=1, \ldots, k$,

$$
\begin{gathered}
\left(E_{i}\right) \quad c_{1} \frac{n-4}{2} \frac{H\left(a_{i}, a_{i}\right)}{\lambda_{i}^{n-4}}+\gamma_{i} c_{1} \sum_{j \neq i} \gamma_{j}\left(\lambda_{i} \frac{\partial \varepsilon_{i j}}{\partial \lambda_{i}}+\frac{n-4}{2} \frac{H\left(a_{i}, a_{j}\right)}{\left(\lambda_{i} \lambda_{j}\right)^{(n-4) / 2}}\right)+\frac{n-4}{2} c_{2} \varepsilon \\
=o\left(\varepsilon+\sum_{j=1}^{k} \frac{1}{\left(\lambda_{j} d_{j}\right)^{n-4}}+\sum_{r \neq j} \varepsilon_{r j}\right) .
\end{gathered}
$$

Observe that, if $j<i$, we have $\lambda_{j}\left|a_{i}-a_{j}\right|$ is bounded (by the assumption) which implies that

$$
\begin{array}{ll}
\left|a_{i}-a_{j}\right|=o\left(d_{j}\right), \quad d_{i} / d_{j}=1+o(1), \quad \forall i, j \quad \text { and } \\
\varepsilon_{i j} \geq c\left(\lambda_{j} / \lambda_{i}\right)^{(n-4) / 2}, \quad \forall j<i,
\end{array}
$$

where $c$ is a positive constant. Using (4.21), easy computations show that

$$
\begin{aligned}
\varepsilon_{(i-1) j}+\varepsilon_{i(j+1)} & =o\left(\varepsilon_{i j}\right), \quad \forall i<j, \\
\frac{H\left(a_{i}, a_{j}\right)}{\left(\lambda_{i} \lambda_{j}\right)^{(n-4) / 2}} & =o\left(\frac{1}{\left(\lambda_{1} d_{1}\right)^{n-4}}\right) \quad \text { if }(i, j) \neq(1,1) .
\end{aligned}
$$

Thus, using (4.22), $\left(E_{i}\right)$ can be written as

$$
\begin{aligned}
& \left(E_{1}^{\prime}\right) \quad c_{1} \frac{n-4}{2} \frac{H\left(a_{1}, a_{1}\right)}{\lambda_{1}^{n-4}}+c_{1} \gamma_{1} \gamma_{2} \lambda_{1} \frac{\partial \varepsilon_{12}}{\partial \lambda_{1}}+\frac{n-4}{2} c_{2} \varepsilon=o\left(\varepsilon+\frac{1}{\left(\lambda_{1} d_{1}\right)^{n-4}}+\sum_{r \neq j} \varepsilon_{r j}\right), \\
& \left(E_{k}^{\prime}\right) \quad c_{1} \gamma_{k-1} \gamma_{k} \lambda_{k} \frac{\partial \varepsilon_{(k-1) k}}{\partial \lambda_{k}}+\frac{n-4}{2} c_{2} \varepsilon=o\left(\varepsilon+\frac{1}{\left(\lambda_{1} d_{1}\right)^{n-4}}+\sum_{r \neq j} \varepsilon_{r j}\right),
\end{aligned}
$$

and for $1<i<k$,

$$
\left(E_{i}^{\prime}\right) \quad c_{1} \gamma_{i-1} \gamma_{i} \lambda_{i} \frac{\partial \varepsilon_{(i-1) i}}{\partial \lambda_{i}}+c_{1} \gamma_{i} \gamma_{i+1} \lambda_{i} \frac{\partial \varepsilon_{i(i+1)}}{\partial \lambda_{i}}+\frac{n-4}{2} c_{2} \varepsilon=o\left(\varepsilon+\frac{1}{\left(\lambda_{1} d_{1}\right)^{n-4}}+\sum_{r \neq j} \varepsilon_{r j}\right) .
$$

The proof will depend on the value of $l$ which is defined in the theorem.

Case 1. $l=k$. From the definition of $l$ we get $\gamma_{k-1} \gamma_{k}=-1$. Now using (4.1) and $\left(E_{k}^{\prime}\right)$, we derive that

$$
\varepsilon=o\left(\frac{1}{\left(\lambda_{1} d_{1}\right)^{n-4}}+\sum_{r \neq j} \varepsilon_{i j}\right) \quad \text { and } \quad \varepsilon_{(k-1) k}=o\left(\frac{1}{\left(\lambda_{1} d_{1}\right)^{n-4}}+\sum_{r \neq j} \varepsilon_{r j}\right) .
$$

Now, using (4.23) and $\left(E_{k-1}^{\prime}\right)$, we derive the estimate of $\varepsilon_{(k-2)(k-1)}$ and by induction we get

$$
\varepsilon_{(i-1) i}=o\left(\frac{1}{\left(\lambda_{1} d_{1}\right)^{n-4}}+\sum_{r \neq j} \varepsilon_{r j}\right) \quad \text { for each } i=2, \ldots, k
$$


Finally, using (4.22), (4.23), (4.24), and $\left(E_{1}^{\prime}\right)$ we obtain

$$
\frac{H\left(a_{1}, a_{1}\right)}{\lambda_{1}^{n-4}}=o\left(\frac{1}{\left(\lambda_{1} d_{1}\right)^{n-4}}\right)
$$

which gives a contradiction.

Case 2. $l=k-1$. Using (4.1), an easy computation implies that

$$
\lambda_{k-1} \frac{\partial \varepsilon_{(k-1) k}}{\partial \lambda_{k-1}}-\lambda_{k} \frac{\partial \varepsilon_{(k-1) k}}{\partial \lambda_{k}} \geq c \varepsilon_{(k-1) k}
$$

Then from $\left(E_{k-1}^{\prime}\right),\left(E_{k}^{\prime}\right),(4.1),(4.25)$, and the fact that $\gamma_{k-1} \gamma_{k}=1$ and $\gamma_{k-2} \gamma_{k-1}=-1$ (since $l=k-1)$, we obtain

$$
c \varepsilon_{(k-1) k}+\varepsilon_{(k-2)(k-1)}=o\left(\varepsilon+\frac{1}{\left(\lambda_{1} d_{1}\right)^{n-4}}+\sum_{r \neq j} \varepsilon_{r j}\right) .
$$

Now using $\left(E_{k}^{\prime}\right)$ and (4.26) we get (4.23) and as before, (4.24) is satisfied. Hence we also derive a contradiction from $\left(E_{1}^{\prime}\right)$.

Case 3. $l \notin\{k, k-1\}$. Recall that in this case we have assumed that $\lambda_{l}\left|a_{l}-a_{l+1}\right| \rightarrow 0$. This implies that

$$
\lambda_{l} \frac{\partial \varepsilon_{l(l+1)}}{\partial \lambda_{l}}=((n-4) / 2) \varepsilon_{l(l+1)}(1+o(1)) .
$$

Hence, using $\left(E_{l}^{\prime}\right)$, the definition of $l$ and (4.1) we obtain the first part of (4.23). The second part follows from $\left(E_{k}^{\prime}\right)$ and the first one. Finally, as before we derive a contradiction from $\left(E_{1}^{\prime}\right)$.

Hence, our theorem is proved.

\section{Competing interests}

The author declares that they have no competing interests.

\section{Acknowledgements}

The author gratefully acknowledges the Deanship of Scientific Research at Taibah University on material and moral support, in particular by financing this research project.

Received: 26 August 2014 Accepted: 1 December 2014 Published: 30 Dec 2014

\section{References}

1. Bartsch, T, Weth, T: A note on additional properties of sign-changing solutions to superlinear elliptic equations. Topol. Methods Nonlinear Anal. 22, 1-14 (2003)

2. Ben Ayed, M, El Mehdi, K, Grossi, M, Rey, O: A nonexistence result of single peaked solutions to a supercritical nonlinear problem. Commun. Contemp. Math. 2, 179-195 (2003)

3. Ben Ayed, M, Ould Bouh, K: Nonexistence results of sign-changing solution to a supercritical nonlinear problem. Commun. Pure Appl. Anal. 7(5), 1057-1075 (2008)

4. Castro, A, Cossio, J, Newberger, JM: A sign-changing solution for a supercritical Dirichlet problem. Rocky Mt. J. Math. 27, 1041-1053 (1997)

5. Del Pino, M, Felmer, P, Musso, M: Two bubbles solutions in the supercritical Bahri-Coron's problem. Calc. Var. Partial Differ. Equ. 16, 113-145 (2003)

6. Ould Bouh, K: Nonexistence result of sign-changing solutions for a supercritical problem of the scalar curvature type. Adv. Nonlinear Stud. 12, 149-171 (2012)

7. Ben Ayed, M, El Mehdi, K: On a biharmonic equation involving nearly critical exponent. Nonlinear Differ. Equ. Appl. 13(4), 485-509 (2006)

8. Ben Ayed, M, Ghoudi, R: Profile and existence of sign-changing solutions to an elliptic subcritical equation. Commun. Contemp. Math. 10(6), 1183-1216 (2008)

9. Chou, KS, Geng, D: Asymptotics of positive solutions for a biharmonic equation involving critical exponent. Differ. Integral Equ. 13, 921-940 (2000) 
10. van der Vorst, RCAM: Fourth order elliptic equations with critical growth. C. R. Acad. Sci. Paris 320, 295-299 (1995)

11. Ebobisse, F, Ould Ahmedou, M: On a nonlinear fourth order elliptic equation involving the critical Sobolev exponent. Nonlinear Anal. TMA 52, 1535-1552 (2003)

12. Gazzola, F, Grunau, HC, Squassina, M: Existence and nonexistence results for critical growth biharmonic elliptic equations. Calc. Var. Partial Differ. Equ. 18, 117-143 (2003)

13. Ould Bouh, K: Sign-changing solutions of a fourth-order elliptic equation with supercritical exponent. Electron. J. Differ. Equ. 2014, 77 (2014)

14. Bahri, A, Coron, JM: On a nonlinear elliptic equation involving the critical Sobolev exponent: the effect of topology of the domain. Commun. Pure Appl. Math. 41, 255-294 (1988)

15. Rey, O: The role of Green's function in a nonlinear elliptic equation involving the critical Sobolev exponent. J. Funct. Anal. 89, 1-52 (1990)

16. Rey, O: The topological impact of critical points at infinity in a variational problem with lack of compactness: the dimension 3. Adv. Differ. Equ. 4, 581-616 (1999)

17. Bahri, A: Critical Point at Infinity in Some Variational Problems. Pitman Res. Notes Math. Ser., vol. 182. Longman, Harlow (1989)

18. Bahri, A, Li, YY, Rey, O: On a variational problem with lack of compactness: the topological effect of the critical points at infinity. Calc. Var. Partial Differ. Equ. 3, 67-94 (1995)

19. Ben Ayed, M, Chtioui, H, Hammami, M: A Morse lemma at infinity for Yamabe type problems on domains. Ann. Inst. Henri Poincaré, Anal. Non Linéaire 20, 543-577 (2003)

10.1186/1687-1847-2014-319

Cite this article as: Ould Bouh: On a fourth order elliptic equation with supercritical exponent. Advances in Difference Equations 2014, 2014:319

\section{Submit your manuscript to a SpringerOpen ${ }^{\circ}$ journal and benefit from:}

- Convenient online submission

- Rigorous peer review

- Immediate publication on acceptance

- Open access: articles freely available online

- High visibility within the field

- Retaining the copyright to your article 